\title{
Structure-Function Relationship of PAMAM Dendrimers as Robust Oil Dispersants
}

\author{
Nicholas K. Geitner, ${ }^{\dagger}$ Bo Wang, ${ }^{\dagger}$ Rachel E. Andorfer, ${ }^{\dagger}$ David A. Ladner, ${ }^{\ddagger}$ Pu Chun Ke, ${ }^{\dagger}$ and Feng Ding ${ }^{*}{ }^{\dagger}$ \\ ${ }^{\dagger}$ Department of Physics and Astronomy and ${ }^{\ddagger}$ Department of Environmental Engineering and Earth Sciences, Clemson University, \\ Clemson, South Carolina 29634, United States
}

\section{Supporting Information}

ABSTRACT: PAMAM dendrimers have recently been investigated as efficient and biocompatible oil dispersants utilizing their encapsulation capacity; however, their high cationic charge density has been shown to be cytotoxic. It is therefore imperative to mitigate cationic charge-induced toxicity and understand the effects of such changes. Presented here is a synergistic experimental and computational approach to examine the effects of varying terminal surface charge on the capacity of dendrimers to disperse model liner, polycyclic aromatic, and hybrid hydrocarbons. Uncharged dendrimers collapse by forming intramolecular hydrogen bonds, which reduce the hosting capability. On the other hand, changing the surface charges from positive to negative greatly shifts the $\mathrm{p} K_{\mathrm{a}}$ of tertiary amines of the PAMAM dendrimer interior. As a result, the negatively charged dendrimers have a significant percentage of tertiary amines protonated, $30 \%$. This unexpected change in the interior protonation state causes electrostatic interactions with the anionic terminal groups, leading to contraction and a marked decrease in hydrocarbon hosting capacity. The present work highlights the robust nature of dendrimer oil dispersion and also illuminates potentially unintended or unanticipated effects of varying dendrimer surface chemistry on their encapsulation or hosting efficacy, which is important for their environmental, industrial, and biomedical applications.

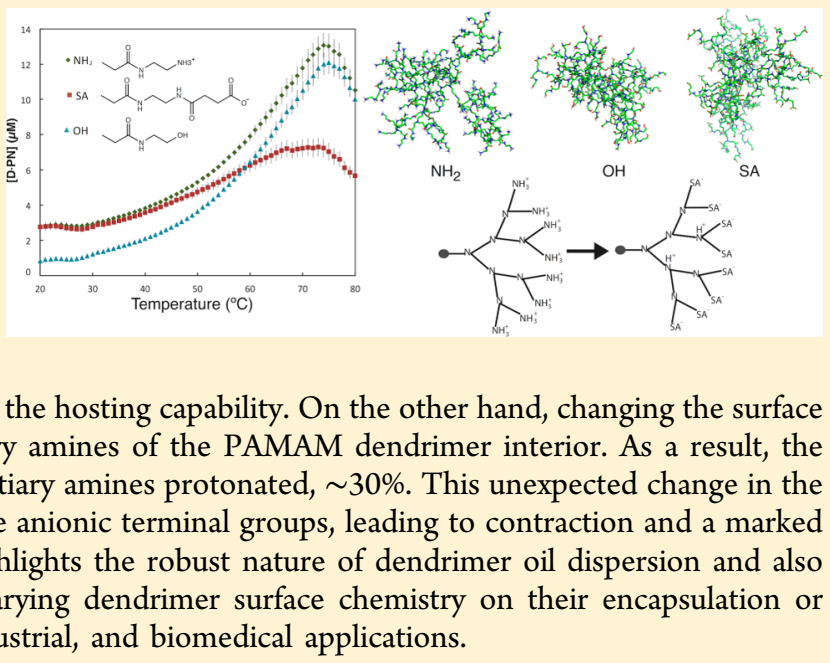

\section{INTRODUCTION}

Originally proposed by Paul Flory, ${ }^{1}$ dendritic polymers are a class of macromolecules consisting of highly branched polymer units. Within this class are dendrons, dendrimers, and hyperbranched polymers. ${ }^{1}$ Dendrimers can be precisely synthesized with high order and monodispersion, with welldefined branching units emanating from a central core. ${ }^{1}$ The number of these branching iterations is termed the generation of the dendrimer and determines its size, structure, and function. Hyperbranched polymers, in contrast, possess less well-defined branched interiors, resulting in a higher polydispersity at a much lower production cost. Due to their unique physicochemical properties, there are a wide variety of current and potential applications of dendrimers ranging from environment to energy and biomedicine. For example, hydroxyl-terminated PAMAM dendrimers have been shown to remove contaminants such as humic acids ${ }^{2}$ and metal ions $s^{3,4}$ from drinking water or contaminated soils. Dendrimers can be used in light-harvesting applications for superior transduction efficiency in diodes and other photonic devices. ${ }^{5,6}$ The surface functionality of PAMAM dendrimers has been altered to include long-lifetime ibuprofen release in vivo, ${ }^{7}$ and conjugation with partially anionic folate-conjugates has been explored for the delivery of antiarthritic drugs. ${ }^{8}$ The ability of dendrimers to encapsulate small organic molecules has also been studied in terms of dendrimer generations ${ }^{9}$ as well as the shape of a guest molecule, ${ }^{10}$ demonstrating a wide array of hosting capabilities of dendrimers in aqueous solution.

Given their hosting capabilities, we have previously proposed PAMAM polymers as oil dispersants ${ }^{11}$ and showed that cationic PAMAM dendrimers are capable of hosting both polyaromatic and linear hydrocarbons in water. ${ }^{11}$ Conventionally, lipid-like oil dispersants have been in use since at least the $1960 \mathrm{~s}^{12}$ and also during the large scale Deepwater Horizon disaster of 2010. However, concerns over the potential toxicity of conventional oil dispersants have been recently raised. ${ }^{13-15}$ There is a renewed and pressing desire for effective yet biocompatible dispersing agents. Our previous work has shown, however, that highly cationic amine-terminated poly(amidoamine) (PAMAM) dendrimers cause acute toxicity in amoebas at a high concentration. ${ }^{16}$ Similarly, several other studies have also shown that highly cationic PAMAM dendrimers cause significant charge-induced toxicity in vitro ${ }^{17-20}$ and rapid blood clotting in vivo. ${ }^{21}$ It has been suggested that the electrostatic interaction between highly cationic PAMAM and negatively charged cell membrane results in pore formation to trigger cytotoxicity. Therefore, efforts are increasingly being focused on altering dendrimer terminal charges in order to

Received: August 5, 2014

Revised: September 25, 2014

Accepted: October 3, 2014

Published: October 3, 2014 
reduce the toxicity or improve the efficacy of dendrimer agents. $^{22,23}$

Many studies have been conducted on the size, structure, and dynamics of dendrimers depending on dendrimer generation $^{24,25}$ and environmental conditions such as solution $\mathrm{pH}$ and ionic strength. ${ }^{24-28}$ It has been shown that PAMAM dendrimers adopt globular-like structures with the repeating monomers loosely packed in the interior and the surface groups protruding, forming hydrogen bonds with water. Simulations revealed dynamically forming pores in the interior that can bind various guest molecules. ${ }^{24,29}$ Solution $\mathrm{pH}$ and ionic strength can also affect dendrimer structure by changing the dendrimer protonation states and screening of electrostatic interactions, respectively. ${ }^{26,27,30}$ It is not understood, however, how surface modifications of dendrimers, a common strategy in dendrimer design and synthesis, might affect their size, structure, dynamics, and subsequent functionality.

Here, we investigate the effects of varying the surface charge and functionality on dendrimers' ability to serve as effective oil dispersants. Specifically, we examine cationic amine-terminated $\left(\mathrm{G} 4-\mathrm{NH}_{2}\right)$, neutral hydroxyl-terminated $(\mathrm{G} 4-\mathrm{OH})$, and anionic succinamic acid-terminated (G4-SA) PAMAM dendrimers (Figure 1A). Synergistic experiments and molecular dynamics
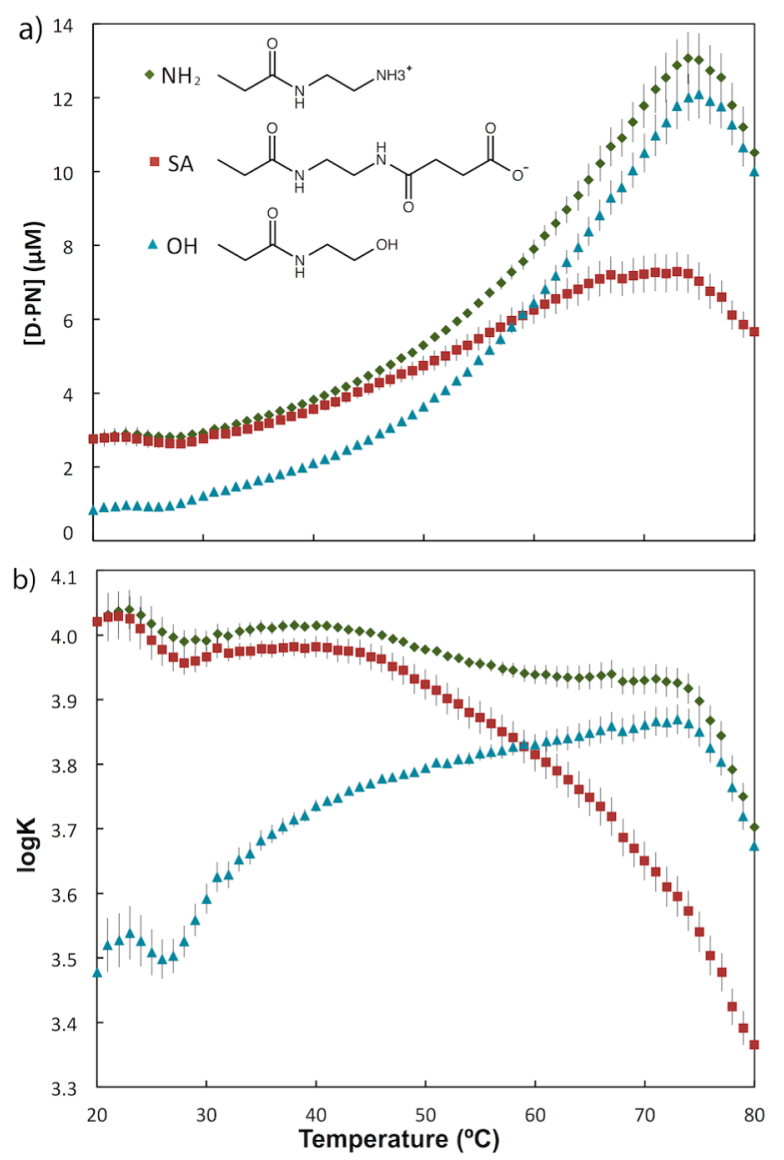

Figure 1. Concentration of dendrimer-associated phenanthrene (a) and the corresponding $\operatorname{logK}$ association constants (b) for G4- $\mathrm{NH}_{2}$ (green diamonds), G4-SA (red squares), and G4-OH (blue triangles). Error bars are standard deviations of 3 independent trials. Note clear transition temperatures at $74{ }^{\circ} \mathrm{C}$ where binding with phenanthrene becomes much less efficient. The inset in (a) shows the chemical structures of a single terminal chain of $\mathrm{G} 4-\mathrm{NH}_{2},-\mathrm{SA}$, and $-\mathrm{OH}$ from top to bottom. simulations are performed to probe the interactions, limitations, mechanisms, and differences between cationic, anionic, and neutrally charged PAMAM dendrimers with linear, polyaromatic, and hybrid hydrocarbons as well as the combination thereof. These various combinations of hydrocarbon are studied in order to gain a more fundamental understanding of dendrimer oil dispersant interactions with the various hydrocarbon components of crude oil as well as illuminate any potential synergistic dispersion effects of hydrocarbon mixtures. The advantages of model hydrocarbons over whole crude oil include the real-time tracking and accurate quantification for mechanistic studies of the structure-function relationship. Additional studies of dendrimer dispersion efficacy and toxicity with crude oil have been done in a separate work. The implications of this study reach beyond oil dispersion to other biomedical and environmental applications including drug delivery and water purification, noting the differences in dendrimer interactions with aliphatic and aromatic hydrophobic molecules as well as potentially unanticipated effects of altering dendrimer surface functionality. We find that marked differences in hosting capacity for hydrocarbons arise from changes in both the structure and dynamics of the dendrimers with varying terminal functionality.

\section{EXPERIMENTAL SECTION}

2.1. Materials and Characterization. All dendrimers were purchased from Dendritech, Inc. and were PAMAM G4.0 (generation four) in water solvent and stored at $4{ }^{\circ} \mathrm{C}$. Phenanthrene (PN) and octadecylbenzene (ODB) were purchased from Sigma-Aldrich, hexadecane $\left(\mathrm{C}_{16}\right)$ was purchased from Acros Organics, and all were stored at room temperature. The dendrimer stock solutions were diluted in DI water $(18 \mathrm{M} \Omega \mathrm{cm})$ to a final concentration of $15 \mu \mathrm{M}$, and their $\mathrm{pH}$ adjusted to 8.2 to mimic that of seawater using $1 \mathrm{M} \mathrm{NaOH}$ and $1 \mathrm{M} \mathrm{HCl}$. Dynamic light scattering (DLS) and zeta potential characterizations of these prepared stock solutions were carried out on a NanoBrook ZetaPALS.

2.2. UV-vis Spectophotometry and Phenanthrene Affinity. UV-vis spectroscopy absorbance measurements were performed on a temperature-controlled Cary 300 Bio (Thermo Electric Corp.). To normalize the concentration of $\mathrm{PN}$, a known quantity was dissolved in methanol and the intensity of the absorbance peak at $251 \mathrm{~nm}$ was measured. This relation was then used to calculate all other PN concentrations. The concentration of dendrimer-associated $\mathrm{PN}$ was calculated using eq 1 where $[\mathrm{PN}]_{\mathrm{T}}$ is the total observed concentration of $\mathrm{PN}$ in the column and $[\mathrm{PN}]_{S}$ is the concentration of free $\mathrm{PN}$ in solution.

$$
[\mathrm{D} \cdot \mathrm{PN}]=[\mathrm{PN}]_{\mathrm{T}}-[\mathrm{PN}]_{\mathrm{S}}
$$

A solution of $15 \mu \mathrm{M}$ dendrimers was used as a control in all measurements of PN with dendrimers. Each sample was prepared with $1 \mathrm{mg}$ of $\mathrm{PN}$ added to $2 \mathrm{~mL}$ of either water or dendrimer stock solution. Samples were bath sonicated for 5 min (Branson) in order to break PN solids and then rotated overnight to reach equilibrium. We then measured the affinity of dendrimers for $\mathrm{PN}$ in water as a function of temperature by measuring the absorbance of $\mathrm{PN}$ over a temperature range from 20 to $80^{\circ} \mathrm{C}$. The temperature was increased at a rate of $0.1{ }^{\circ} \mathrm{C} /$ min, and absorbance was measured every $1.0 \pm 0.02{ }^{\circ} \mathrm{C}$. These measurements were made in triplicate in sealed quartz cuvettes. The apparent association constant $K$ was calculated using eq 2 where $[\mathrm{D}]$ is the free dendrimer concentration. 


$$
K=[\mathrm{D} \cdot \mathrm{PN}] /\left([\mathrm{D}][\mathrm{PN}]_{\mathrm{S}}\right)
$$

Solutions of PN dissolved in $\mathrm{C}_{16}$ were prepared such that the final solution was $8 \% \mathrm{PN}$ by weight dissolved in $\mathrm{C}_{16}$. For sample incubations, $20 \mu \mathrm{L}$ of this stock was added to $2 \mathrm{~mL}$ of either water or dendrimer solution and then rotated for $1 \mathrm{~h}$. This ensured that the same total mass of $\mathrm{PN}$ was added as in the pure $\mathrm{PN}$ experiments. The same temperature ramp as above was then performed, again by measuring the absorbance of PN at $251 \mathrm{~nm}$.

2.3. Fluorescence. Fluorescence measurements were performed on a temperature-controlled Cary Eclipse fluorometer (Thermo Electric Corp.). ODB-doped $\mathrm{C}_{16}$ stock was prepared such that the hydrocarbon solution was $2.6 \%$ ODB by weight. For all measurements with $\mathrm{ODB}, 65 \mu \mathrm{L}$ of stock solution was added to $2 \mathrm{~mL}$ of water or dendrimer solution and then rotated gently for $1 \mathrm{~h}$. It was then allowed to settle, and solution was pulled from the middle of each tube to avoid phase-separated oil. Then $20 \mu \mathrm{L}$ of stock ODB-doped $\mathrm{C}_{16}$ was added to each cuvette to ensure a consistent excess of available hydrocarbons. The fluorescence emission was observed at both wavelengths of 290 and $299 \mathrm{~nm}$, with an excitation wavelength of $258 \mathrm{~nm}$ in both cases. The fluorescence emission intensities were recorded every $1.0 \pm 0.02{ }^{\circ} \mathrm{C}$ in the same temperature ramp as described in section 2.2 .

2.4. DMD Simulations. Discrete molecular dynamics (DMD) is a special type of molecular dynamics algorithm, featuring rapid dynamics sampling efficiency. The detailed algorithm and force field parametrization of DMD can be found elsewhere. ${ }^{31}$ We used a united atom representation to model the molecular system, explicitly modeling all polar hydrogen and heavy atoms and with implicit solvent. Interatomic interactions were modeled by a physical force field adapted from Medusa, ${ }^{32,33}$ which included van der Waals (VDW), solvation, electrostatic, and hydrogen bond interactions. The force field parameters for VDW interactions, bond length, angle, and dihedrals were taken from CHARMM 19. ${ }^{34}$ The solvation energy was included using the Lazaridis-Karplus implicit solvent model. ${ }^{35}$ The distance and angular dependent hydrogen bond interaction was modeled using a reaction-like algorithm. ${ }^{33}$ We used the Debye-Hückel approximation to model the screened electrostatic interactions between charged atoms. The Debye length was approximately $10 \AA$ by assuming water relative permittivity of 80 and a monovalent electrolyte concentration of $0.1 \mathrm{M}$.

The starting structures of dendrimers were generated by constructing the idealized 3-dimensional dendrimer structure consisting of a core, branching units, and terminal groups, followed by equilibration and energy minimization. To emulate a solution $\mathrm{pH}$ of 8.2 , all G4- $\mathrm{NH}_{2}$ and G4-SA terminal groups were charged (protonated and deprotonated, respectively). All tertiary amines in $\mathrm{G} 4-\mathrm{NH}_{2}$ and $\mathrm{G} 4-\mathrm{OH}$ were deprotonated and therefore uncharged. To model the partial protonation of tertiary amines in the presence of acid terminal groups, the protonation state of the interior tertiary amines of G4-SA was varied, where $0,10,20$, or $30 \%$ of randomly selected tertiary amines were protonated. In our simulations, the net charges of the molecular systems were maintained zero by adding offsetting charges, such as chloride $\left(\mathrm{Cl}^{-}\right)$and sodium $\left(\mathrm{Na}^{+}\right)$ ions. After the initialization of dendrimer structures, energy minimization using DMD was carried out for 10,000 time steps (approximately $10 \mathrm{~ns}$ ) before carrying out further equilibrium simulations.
In DMD simulations, temperature is in the unit of kcals $/ \mathrm{mol}$. $k_{\mathrm{B}}$, where $k_{\mathrm{B}}$ is the Boltzmann constant. Our simulations were conducted for a temperature range of $0.55-0.75 \mathrm{kcal} / \mathrm{mol} \cdot k_{\mathrm{B}}$, corresponding approximately to $275-375 \mathrm{~K}$. The Anderson's thermostat $^{36}$ was used to perform constant temperature simulations. At each temperature, energy minimization was first carried out for $10 \mathrm{~ns}$, and the simulations were conducted for 2 million time steps (approximately $1 \mu \mathrm{s}$ ), corresponding to an average of approximately $72 \mathrm{CPU}$ h. We characterized the sizes of all three types of dendrimers by measuring the radius of gyration $\left(R_{\mathrm{g}}\right)$ as a function of temperature. The mean and standard deviation of $R_{\mathrm{g}}$ were obtained from 8,000 snapshots evenly distributed throughout the final $800 \mathrm{~ns}$ of simulation.

\section{RESULTS AND DISCUSSION}

Distinctive Physicochemical Properties of Dendrimers with Modified Terminal Groups. Generation 4 PAMAM dendrimers of positively $\left(\mathrm{NH}_{2}\right)$, negatively (SA), and neutrally $(\mathrm{OH})$ charged functional groups, all at $\mathrm{pH}$ 8.2, were first incubated with $\mathrm{PN}$. We measured the concentration of saturated PN in water and in dendrimer solution with an excess of PN (see eq 1, section 2.2 in the Experimental Section; Figure S1) and computed the concentration of dendrimerassociated PN [D.PN]. We determined the [D.PN], quantifying the capacity of dendrimer to host $\mathrm{PN}$, as a function of temperature (Figure 1a). The temperature range of $20-80{ }^{\circ} \mathrm{C}$ was chosen to examine the fundamental differences in dendrimer behavior and interactions with hydrocarbons at environmentally relevant temperatures and beyond. Initially, the positively and negatively charged dendrimers have similar hosting capacities, while the neutrally charged dendrimer has lower hosting capacity. As temperature increases in all cases there is an increased hosting of PN by dendrimers, in part due to the increasing availability of PN in solution with increasing temperature. This trend continues until approximately $74{ }^{\circ} \mathrm{C}$ for positively and neutrally charged dendrimers, at which point the PN hosting capacity reaches a peak followed by a marked decrease. In contrast, negatively charged dendrimers reach their maximum capacity between 65 and $74{ }^{\circ} \mathrm{C}$, reaching just $56 \%$ the maximum $\mathrm{PN}$ hosting of G4- $\mathrm{NH}_{2}$.

With the measured PN concentrations in water and in dendrimers as well as the concentration of dendrimers in solution, we can calculate the apparent association constants, $K$ (see eq 2, section 2.2 in the Experimental Section), and compute $\log K$ as a function of temperature (Figure $1 \mathrm{~b}$ ). For $\mathrm{NH}_{2}$ and SA-terminated dendrimers, we observe relatively constant, large apparent association constants at low temperatures. In contrast, the neutral OH-terminated dendrimers had a much lower affinity at low temperature, but this affinity surprisingly increases rapidly with respect to increased temperature and becomes nearly identical to the $\mathrm{NH}_{2}$ terminated affinity near $70{ }^{\circ} \mathrm{C}$. Both G4- $\mathrm{NH}_{2}$ and G4-OH dendrimer affinity for PN sharply drop at $74{ }^{\circ} \mathrm{C}$, as expected (see Figure 1a). Despite the more significant and gradual decrease in G4-SA affinity, we note an increase in this rate of decrease at the same $74^{\circ} \mathrm{C}$, indicating the temperature at which it becomes thermodynamically more favorable for PN to dissolve in water than to be partitioned inside of the dendrimers, as PN water solubility increases exponentially with temperature over the observed range (Figure S1). Therefore, the changes in dendrimer surface charge result in drastic changes in its hosting capacity of $\mathrm{PN}$ and the temperature dependences. However, since PN is noncharged 
and the binding is not governed by electrostatic interactions, it is intriguing as what the molecular mechanism is for such drastic changes in hydrocarbon hosting capacity upon adjusting the dendrimer surface charges.

We postulated that the changes are mostly in the structure of dendrimer, which in turn affect the hosting function of dendrimer. We first characterized the size and charge properties of all three types of dendrimers in solution (Table 1) using

Table 1. Characterization of PAMAM Dendrimers ${ }^{a}$

\begin{tabular}{ccc} 
functionality & $D_{\mathrm{H}}(\mathrm{nm})$ & \multicolumn{1}{c}{$\zeta(\mathrm{mV})$} \\
\hline $\mathrm{NH}_{2}$ & $4 \pm 1$ & $30.7 \pm 2.9$ \\
$\mathrm{OH}$ & $3 \pm 1$ & $-0.9 \pm 1.0$ \\
$\mathrm{SA}$ & $4 \pm 1$ & $-18.5 \pm 2.0$
\end{tabular}

${ }^{a} D_{\mathrm{H}}$ : hydrodynamic diameter. $\zeta$ : zeta potential.

DLS and PALS zeta potential measurements, respectively (Experimental Section). The DLS results suggest that the dendrimers are fairly monodisperse and tend not to aggregate in DI water. Second, that the $\mathrm{OH}$-terminated dendrimers have smaller hydrodynamic diameters $\left(D_{\mathrm{H}}\right)$ than their charged counterparts. The zeta $(\zeta)$ potential quantifies the dendrimer net electrokinetic potential in solution. We find that, indeed, the $\mathrm{OH}$-terminated dendrimers carry nearly zero net charge and the amine-terminated dendrimers are highly positively charged $(+30 \mathrm{mV})$. Interestingly, the SA-terminated, while negatively charged, carry a net charge with significantly smaller magnitude than the amine-terminated. This reduction of overall net charge suggests that some of the interior tertiary amines in SA-terminated dendrimers may become protonated at this $\mathrm{pH}$. Assuming electric multilayers similar in nature, the measured differences in zeta potential magnitude suggest the protonation of approximately $30 \%$ of G4-SA tertiary amines. Such a significant shift in $\mathrm{p} K_{\mathrm{a}}$ of the tertiary amine compared to neutral and positively charged dendrimers is feasible in the presence of a large number of terminal acidic groups in the vicinity. ${ }^{37}$ These characterizations suggest significant physicochemical differences in PAMAM dendrimers caused simply by varying the terminal functionality. Next, we perform molecular dynamics simulations to study the changes of dendrimer size and structure with respect to surface charges at the molecular level.

We performed DMD simulations of all three dendrimer classes (Experimental Section) and measured the radius of gyration $\left(R_{\mathrm{g}}\right)$ as a function of temperature (Figure $2 \mathrm{a}$ ) for each case. For the SA-terminated dendrimers, we studied the effect of partial protonation of their tertiary amines, with levels of protonation ranging from 0 to $30 \%$ protonation, where $30 \%$ tertiary protonation corresponds to the experimentally observed zeta potential of G4-SA. In DMD simulations, the dendrimer rapidly reaches equilibrium with $R_{\mathrm{g}}$ fluctuating around its average value in a long time scale simulation trajectory ( $\sim 50 \mathrm{~ns}$; Figure S2). Across the simulated temperature range, the $R_{\mathrm{g}}$ of $\mathrm{G} 4-\mathrm{NH}_{2}$ increases from 19.4 to $21.25 \AA$, in agreement with small angle neutron scattering (SANS) experiments as well as atomistic molecular dynamics (MD) and coarse-grained (CG) simulations performed elsewhere (Table 2, Figure S3). ${ }^{38-43}$ Because $R_{\mathrm{g}}$ is an averaged single-value measurement, we also calculate the radial density function (RDF) to quantify the internal structure of dendrimers (Figures S4 and S5). As observed previously in an all-atom MD simulations, ${ }^{43}$ we find that lower generations G2-G3 exhibit more compact core structures while higher generations G4-G5
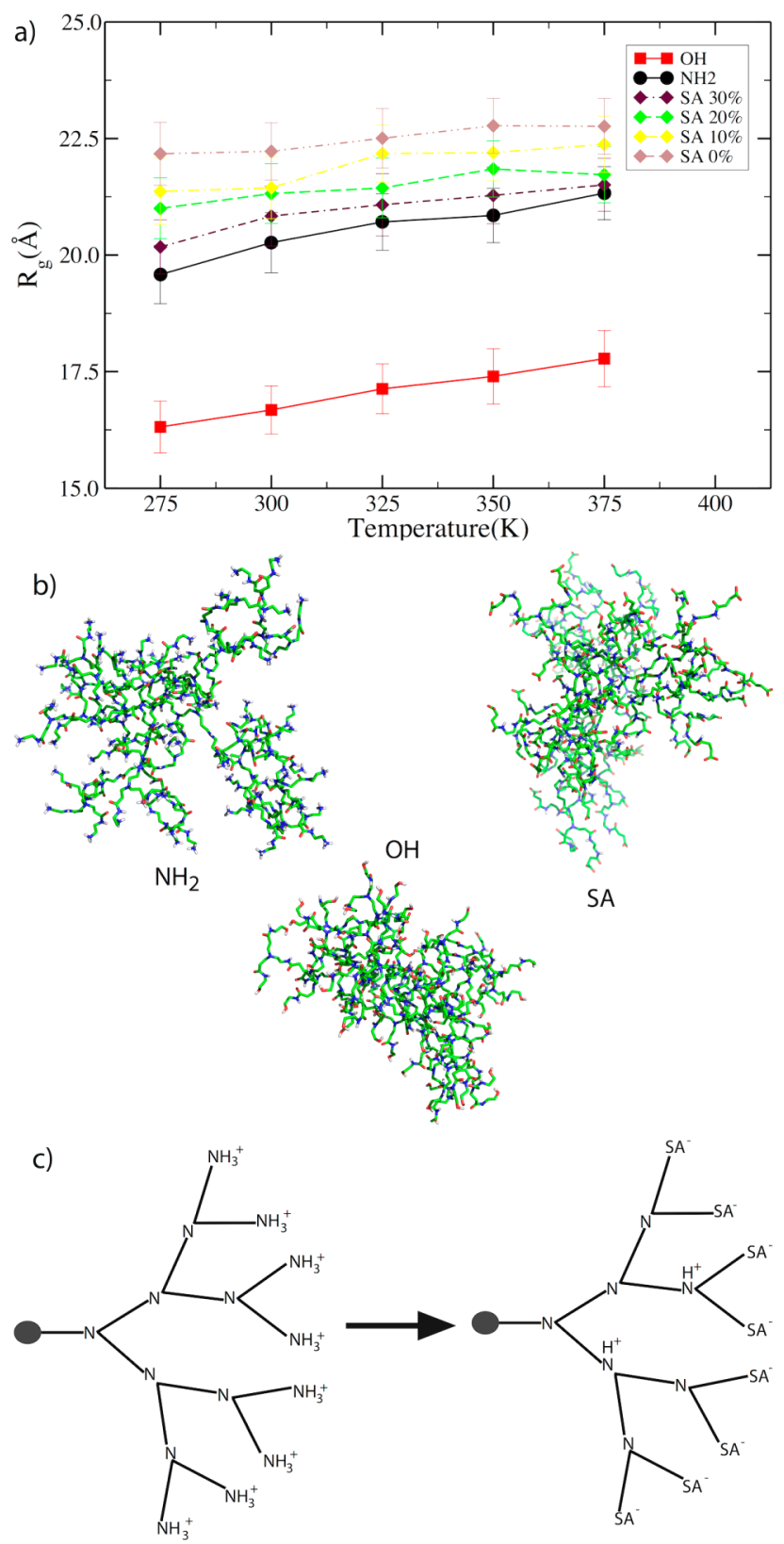

Figure 2. Radius of gyration $\left(R_{\mathrm{g}}\right)$ for $\mathrm{G} 4-\mathrm{NH}_{2}, \mathrm{OH}$, and SA for SA tertiary amine protonation fractions of $0-30 \%$ (a). Error bars are standard deviations taken across the 800 snapshots used in $R_{\mathrm{g}}$ calculations. Representative DMD snapshots of G4 PAMAM dendrimers at room temperature (b), which illustrates the effect of changing terminal group chemistry on the overall structure of the dendrimer. Differences in structure between G4- $\mathrm{NH}_{2}$ and G4-SA are due to partial protonation of SA tertiary amines (c), with a portion of the dendrimer structure shown schematically emanating from a central core.

are more open due to increased electrostatic repulsion between terminal groups (Figure S4). We also computed the RDF for the $\mathrm{G} 4-\mathrm{NH}_{2}$ dendrimers at different $\mathrm{pH}$ values. At high $\mathrm{pH}$, the primary amines are fully deprononated, making the dendrimer neutrally charged. At low $\mathrm{pH}$, the tertiary amines are protonated, and the dendrimer is fully charged. Our results confirm the expected transition from dense-core at high $\mathrm{pH}$ to dense-shell configuration at neutral and low $\mathrm{pH}$ as observed in previous all-atom MD simulations (Figure S5). ${ }^{41}$ These 
Table 2. Comparison of $R_{\mathrm{g}}$ in PAMAM Dendrimers from Various Works

\begin{tabular}{|c|c|c|c|}
\hline & \multicolumn{3}{|c|}{$R_{\mathrm{g}}(\AA)$} \\
\hline & G3 & G4 & G5 \\
\hline Liu et al. - SANS ${ }^{40}$ & $16.7 \pm 1.2$ & $21.4 \pm 0.4$ & $26.8 \pm 0.4$ \\
\hline Lee and Larson - $\mathrm{CG}^{42}$ & $13.1 \pm 0.1$ & & $23.2 \pm 0.1$ \\
\hline Liu et al. - $\mathrm{MD}^{41}$ & $15.8 \pm 0.3$ & $20.6 \pm 0.2$ & $25.3 \pm 0.1$ \\
\hline Yang and da Rocha - $\mathrm{MD}^{43}$ & $15.0 \pm 0.9$ & $21.8 \pm 0.8$ & $23.8 \pm 0.2$ \\
\hline this work $(300 \mathrm{~K})$ & $15.7 \pm 0.6$ & $20.2 \pm 0.6$ & $25.7 \pm 0.4$ \\
\hline
\end{tabular}

benchmark results validate our DMD simulations as efficient and robust for studies of dendrimer structure and dynamics. G4-SA is, across the simulated temperature range, larger than G4- $\mathrm{NH}_{2}$ due to the slightly longer terminal groups. Their $R_{\mathrm{g}}$ values decrease with increasing tertiary amine protonation, and at the lowest tested temperature it reduces from approximately $22.25 \AA$ at $0 \%$ protonation to just $20.0 \AA$ once $30 \%$ of the interior tertiary amines have been protonated. This size change is because of the electrostatic attraction between these protonated groups and the negatively charged terminal carboxyl groups. This attraction also limits the expansion of G4-SA with temperature: e.g. G4-SA (30\%) swells just 1.25 A compared to a $1.9 \AA$ growth seen in G4- $\mathrm{NH}_{2}$, resulting in equal $R_{\mathrm{g}}$ values at the highest temperature in simulations.

While amine- and SA-terminated dendrimers have similar sizes across the entire temperature range, $\mathrm{G} 4-\mathrm{OH}$ is clearly smaller than its charged counterparts, expanding from an $R_{\mathrm{g}}$ of 16.25 to $18.13 \AA$ at the lowest and highest simulation temperatures, respectively. This markedly smaller size is due to the lack of electrostatic repulsion between terminal groups and hydrogen bond formation between the terminal hydroxyl groups, resulting in a much more compact dendrimer structure (e.g., typical snapshot structures in Figure 2b).

These differences in size and how sizes change with temperature in simulations is consistent with the experimentally observed differences in apparent affinity for PN as in Figure 1. G4-OH has a much lower affinity for PN at low temperatures because, at those temperatures, they are significantly more compact than either G4-SA or $-\mathrm{NH}_{2}$, thus reducing the size and accessibility of the interior voids to host $\mathrm{PN}$ as illustrated by the dense-core structure of the neutrally charged dendrimer (Figure S4). As temperature increases, the G4-OH expands with increased $R_{\mathrm{g}}$ by breaking the hydrogen bonds, thereby granting access to its growing interior cavities. Our zeta-potential characterization of $\mathrm{G} 4-\mathrm{NH}_{2}$ and G4-SA suggests that approximately $30 \%$ of the G4-SA tertiary amines are protonated (Figure 2c) assuming tertiary amines in $\mathrm{G} 4-\mathrm{NH}_{2}$ are not protonated. $^{22}$ This change allows strong electrostatic interaction between terminal groups and the protonated tertiary amines, which causes the dendrimer to contract relative to the less protonated G4-SA dendrimers. Such strong electrostatic interaction also noticeably inhibits size expansion with temperature in contrast to the weaker hydrogen bond interaction in G4-OH (Figure 2a). As a result, the G4-SA features a lower host capacity and apparent affinities for PN compared to G4-NH $\mathrm{N}_{2}$. These differences in swelling behavior highlight why the temperature dependence in PN hosting capacity is different for each dendrimer despite all three functionalizations growing with increasing temperature. Such a dependence on hydrophobic core accessibility for hosting small hydrophobic molecules is in agreement with earlier studies by Tomalia et al. with lipophilic dye encapsulation by dendrimers of various generations. ${ }^{9}$ In addition, since the dendrimer volume available for hosting increases rapidly as the cubic power of the size, a small change in $R_{\mathrm{g}}$ (Figure 2) leads to large changes in hosting capacity (Figure 1 ). It is also important to note that, by charging a fraction of the interior groups, the interior voids become slightly less hydrophobic and thus less favorable for hydrocarbon interactions. Another interesting observation in experiments is the sharp decrease of $\mathrm{PN}$ binding at $74{ }^{\circ} \mathrm{C}$ for all dendrimers (Figure 1). We hypothesize that this phenomenon is due to the intrinsic structural properties of dendrimers at different temperatures. As the dendrimers expand with increasing temperature (Figure 2), the cooperative binding with $\mathrm{PN}$ due to interactions among amidoamine monomers is reduced. At high temperatures, the binding is dominated by the interaction between $\mathrm{PN}$ and amidoamine monomer. Therefore, the transition at $74{ }^{\circ} \mathrm{C}$ is the result of dissociation of PN from amidoamine monomer to the solution.

Hosting of Various Classes of Hydrocarbons and Their Mixtures. Having examined the differences between dendrimers of different surface charge, we are now interested in binding between PAMAM dendrimers, using $\mathrm{G} 4-\mathrm{NH}_{2}$ as our model, and different hydrocarbons. Amine-terminated dendrimers were chosen because they exhibited the strongest binding with hydrocarbons across the tested temperature range and therefore allowed the best characterization of the differences between PAMAM binding with different classes of hydrocarbons. As crude oils are composed largely of aliphatic hydrocarbons, it is critical to understand dendrimer interactions with such linear hydrocarbons. However, purely aliphatic hydrocarbons are difficult to track quantitatively in solution. To overcome this difficulty, we doped solutions of hexadecane with octadecylbenzene (ODB, $2.5 \mathrm{w} / \mathrm{w} \%$ ), which is an 18carbon chain with the addition of a benzene ring on one end. The result is a solution with minimal change from a purely aliphatic hydrocarbon mixture but which can be monitored in real time in solution using spectrofluorescence measurements (Figure 3). We characterized the excitation and emission of ODB-doped $\mathrm{C}_{16}$ in various conditions: dissolved in $100 \%$ methanol, suspended as an oil-in-water emulsion in DI water, and in a DI solution of $15 \mu \mathrm{M} \mathrm{G4}-\mathrm{NH}_{2}$ dendrimers (Figure 3a). We note that the emission peak redshifts from 281 to $290 \mathrm{~nm}$ when suspended in water compared to in methanol, which we attribute to an increased polarity of the fluorophore environment. The ODB emission further redshifts to $299 \mathrm{~nm}$ upon incubation with dendrimers, indicating that a significant fraction of ODB molecules interacted directly with $\mathrm{G} 4-\mathrm{NH}_{2}$ rather than simply being suspended in smaller droplets of $\mathrm{C}_{16}$. We measured the kinetics of this fluorescence over time, monitoring ODB emissions at 290 and $299 \mathrm{~nm}$ for pure water and dendrimer solution samples, respectively (Figure $3 \mathrm{~b}$ ). While the ODB fluorescence in water and with dendrimers began with nearly identical intensity, there was a marked initial decrease in water-suspended intensity, a loss of approximately $30 \%$. This indicates that many of the emulsion droplets in the water suspensions quickly coalesced before the final stable emulsion was achieved. Even after this relatively stable emulsion was formed, there is a slow $(1.4 \% / \mathrm{h})$ continued coalescence and a resulting phase separation of the oil-in-water emulsion. Such coalescence is not seen in the dendrimer solution over the observed time period, confirming that such suspensions are more stable than the oil-in-water emulsions. Based on this fluorescence measurement, the stable suspensions formed with $\mathrm{G} 4-\mathrm{NH}_{2}$ at room temperature accommodate $57 \pm$ 

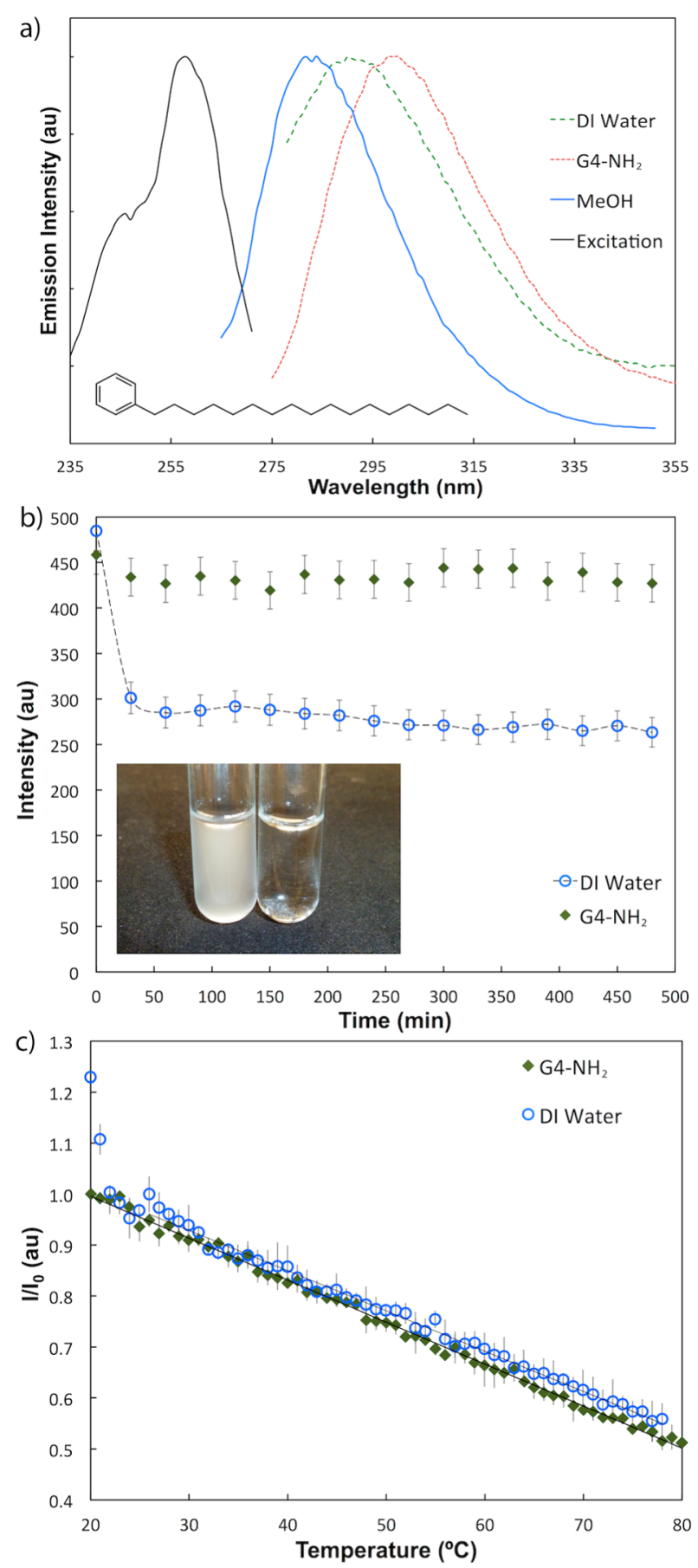

Figure 3. Fluorescence excitation and emission spectra (a) of ODB and the kinetics of this fluorescence at room temperature (b). Inset is a photo of pure water and dendrimer solutions incubated with equal quantities of $\mathrm{C}_{16}+\mathrm{ODB}$. Oil-in-water emulsions are cloudy suspensions (left); dispersion by dendrimers results in a clear suspension (right). Raising the solution temperature causes thermal quenching with and without dendrimers (c) but no loss of binding to dendrimers at high temperatures. Error bars are standard deviations of 3 independent trials.

$4 \%$ more ODB-doped $\mathrm{C}_{16}$ than the oil-in-water emulsion, highlighting the efficiency of dendrimers as oil dispersants. We also note that the nature of the oil dispersion is different from an oil-in-water emulsion (inset, Figure 3b). The oil-in-water emulsion (left) is cloudy due to light scattering by large oil droplets, while the dendrimer-dispersed oil (right) is clear, indicating the presence of much smaller complexes in agreement with previous results that showed the formation of dispersed $\mathrm{C}_{16}$-dendrimer complexes of approximately 200 nm. ${ }^{11}$ This further suggests that nearly all suspended hydrocarbons are dendrimer-associated, since we did not observe any oil-in-water droplets that would be expected if dendrimers simply added encapsulated hydrocarbons to an oilin-water emulsion.

We also examined the fluorescence behavior in water and dendrimer suspensions as a function of temperature (Figure $3 c)$, showing normalized fluorescence intensities. Note that the initial drop in water suspension fluorescence intensity is due to the coalescence observed at early times as in Figure $3 \mathrm{~b}$ but not due to the increase in temperature. Therefore, normalization for the water curve was performed after this initial drop in intensity. After this point, the water and dendrimer suspensions are statistically identical, and both intensities decrease linearly with increasing temperature. This linear decrease in fluorescence intensity with respect to increasing temperature indicates simple thermal quenching as more rotational and vibrational degrees of freedom become accessible, which is different from the molecular quenching observed between cationic dyes and PAMAM dendrimers with organic moieties. ${ }^{44}$ We did not observe any transition as was seen in incubation of pure PN with dendrimers. This is primarily due to the fact that $\mathrm{C}_{16}$ has near zero water solubility, therefore eliminating the competition with water solvation seen in the case of $\mathrm{PN}$ dendrimer interactions. Because of this lack of competition, the $\mathrm{C}_{16}$-dendrimer interactions are more stable at high temperatures.

Because crude oil is a combination of aliphatic and aromatic hydrocarbons (among other components), we created a "model crude" by dissolving $\mathrm{PN}$ in $\mathrm{C}_{16}(8 \% \mathrm{PN})$ to investigate the interaction between $\mathrm{G} 4-\mathrm{NH}_{2}$ dendrimers and hydrocarbon mixtures. By measuring the UV absorbance of PN as described above, the quantity of oil suspended in the water column with and without dendrimers was calculated (Figure 4). In contrast to the trend seen when incubated with pure $\mathrm{PN}$, the dendrimerassociated PN remains approximately constant with temperature across the entire tested temperature range. These results

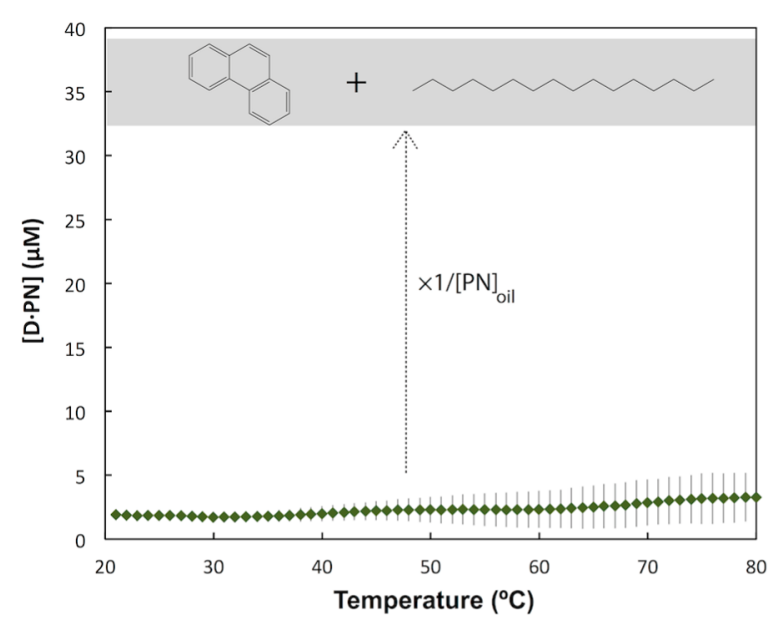

Figure 4. Concentration of dendrimer-associated PN (green diamonds) as a function of temperature. Shaded region shows the calculated increase in concentration of hydrocarbons due to dendrimers compared to oil-in water emulsion, including both $\mathrm{PN}$ and $\mathrm{C}_{16}$. Error bars are standard deviations of 3 independent trials. 
suggest that the aliphatic $\mathrm{C}_{16}$ is able to synergistically facilitate stronger, more stable interactions between dendrimers and $\mathrm{PN}$ that have little temperature sensitivity. We hypothesize that $C_{16}$ accomplishes this by eliminating the $\mathrm{PN}$ partition competition from water solvation, serving as a stronger solvent inside the dendrimer interior for PN. By assuming that the ratio of PN/ $\mathrm{C}_{16}$ remains constant after interacting with dendrimers, we calculated the total suspended hydrocarbon concentration. The increase in this total hydrocarbon concentration compared to that in water alone is shown by the shaded area, reaching at least $35 \mu \mathrm{M}$ hydrocarbon compared to $\sim 10 \mu \mathrm{M}$ of pure PN (Figure 1a); the total concentration of hydrocarbons with dendrimers in water reached approximately $135 \mu \mathrm{M}$. Because of the behavior noted in the ODB- $\mathrm{C}_{16}$ study, we expect that virtually all of the suspended $\mathrm{PN}$ and $\mathrm{C}_{16}$ were directly dendrimer-associated, which indicates a strong hosting capacity of at least 9 hydrocarbons per dendrimer. This capacity for suspending hydrocarbons persisted well beyond environmentally relevant temperatures and indeed even beyond the dissociation temperature for pure PN to break down hydrophobic interaction and $\pi$ stacking.

In summary, we have shown that aliphatic, aromatic, and hybrid hydrocarbons bind strongly with G4 PAMAM dendrimers at environmentally relevant temperatures. Mixtures of aliphatic and aromatic hydrocarbons in a model crude are synergistically dispersed by PAMAM dendrimers, reaching a highly stable dispersion of at least 9 hydrocarbon molecules per G4 dendrimer over a wide range of temperatures. At environmentally relevant temperatures (i.e., less than approximately $32{ }^{\circ} \mathrm{C}$ ), G4-SA and $\mathrm{G} 4-\mathrm{NH}_{2}$ bind much more strongly to hydrocarbons than $\mathrm{G} 4-\mathrm{OH}$ due to this neutral dendrimer collapsing, closing off access to the hydrophobic interior. However, changes in tertiary amine $\mathrm{p} K_{\mathrm{a}}$ and resulting interior protonation in G4-SA due to the abundance of terminal acidic groups severely limited their hydrocarbon hosting capacities. The dendrimer oil dispersions were also shown to be significantly more stable and contained $57 \%$ more hydrocarbon than simple oil-in-water emulsions. These results demonstrate that, when their versatile physicochemical properties are utilized properly, dendrimers are very robust as oil dispersants; we have also illuminated potentially unanticipated or unintended effects of varying dendrimer surface functionality on hosting applications including dispersion but also drug delivery and water purification that usually deal with hydrophobic or charged ligand species. Future work will include studies examining the effects of $\mathrm{pH}$, ionic strength, and ions of different valences in solution.

\section{ASSOCIATED CONTENT}

\section{S Supporting Information}

Figures S1-S5. This material is available free of charge via the Internet at http://pubs.acs.org.

\section{AUTHOR INFORMATION}

\section{Corresponding Author}

*E-mail: fding@clemson.edu.

\section{Notes}

The authors declare no competing financial interest.

\section{ACKNOWLEDGMENTS}

This research was supported in part by US EPA grant RD835182 (to D.L. and F.D.), NSF CBET-1232724 (to
P.C.K and F.D.), and Clemson University startup funds (to F.D.). The simulations were performed on the Palmetto high performance cluster, which is managed and maintained by Clemson University CCIT.

\section{REFERENCES}

(1) Tomalia, D. A.; Fréchet, J. M. J. Discovery of Dendrimers and Dendritic Polymers: A Brief Historical Perspective. J. Polym. Sci., Part A: Polym. Chem. 2002, 40, 2719-2728.

(2) Bhattacharya, P.; Conroy, N.; Rao, A. M.; Powell, B. A.; Ladner, D. A.; Ke, P. C. PAMAM Dendrimer for Mitigating Humic Foulant. RSC Adv. 2012, 2, 7997.

(3) Diallo, M. S.; Balogh, L.; Shafagati, A.; Johnson, J. H.; Goddard, W. A., III; Tomalia, D. A. Poly (amidoamine) Dendrimers: A New Class of High Capacity Chelating Agents for Cu (II) Ions. Environ. Sci. Technol. 1999, 33, 820-824.

(4) Xu, Y.; Zhao, D. Removal of Copper from Contaminated Soil by Use of Poly(amidoamine) Dendrimers. Environ. Sci. Technol. 2005, 39, 2369-2375.

(5) Bradshaw, D. S.; Andrews, D. L. Mechanisms of Light Energy Harvesting in Dendrimers and Hyperbranched Polymers. Polymers (Basel) 2011, 3, 2053-2077.

(6) Nantalaksakul, A.; Reddy, D. R.; Bardeen, C. J.; Thayumanavan, S. Light Harvesting Dendrimers. Photosynth. Res. 2006, 87, 133-150.

(7) Kurtoglu, Y. E.; Mishra, M. K.; Kannan, S.; Kannan, R. M. Drug Release Characteristics of PAMAM Dendrimer-Drug Conjugates with Different Linkers. Int. J. Pharm. 2010, 384, 189-194.

(8) Chandrasekar, D.; Sistla, R.; Ahmad, F. J.; Khar, R. K.; Diwan, P. V. The Development of Folate-PAMAM Dendrimer Conjugates for Targeted Delivery of Anti-Arthritic Drugs and Their Pharmacokinetics and Biodistribution in Arthritic Rats. Biomaterials 2007, 28, 504-512.

(9) Watkins, D. M.; Sayed-sweet, Y.; Klimash, J. W.; Turro, N. J.; Tomalia, D. A. Dendrimers with Hydrophobic Cores and the Formation of Supramolecular Dendrimer - Surfactant Assemblies. Langmuir 1997, 13, 3136-3141.

(10) Jansen, J. F. G. A.; Meijer, E. W.; de Brabander-van den Berg, E. M. M. The Dendritic Box: Shape-Selective Liberation of Encapsulated Guests. J. Am. Chem. Soc. 1995, 117, 4417-4418.

(11) Geitner, N. K.; Bhattacharya, P.; Steele, M.; Chen, R.; Ladner, D. A.; Ke, P. C. Understanding Dendritic Polymer-hydrocarbon Interactions for Oil Dispersion. RSC Adv. 2012, 2, 9371.

(12) Board, C. on E. of O. D. M. Using Oil Spill Dispersants on the Sea; National Academy Press: Washington, DC, 1989; p 352.

(13) Anderson, S. E.; Franko, J.; Lukomska, E.; Meade, B. J. Potential Immunotoxicological Health Effects Following Exposure to COREXIT 9500A during Cleanup of the Deepwater Horizon Oil Spill. Science (80-.) 2011, 74, 1419-1430.

(14) Goodbody-Gringley, G.; Wetzel, D. L.; Gillon, D.; Pulster, E.; Miller, A.; Ritchie, K. B. Toxicity of Deepwater Horizon Source Oil and the Chemical Dispersant, Corexit ${ }^{\circledR}$ 9500, to Coral Larvae. PLoS One 2013, 8, e45574.

(15) Hemmer, M. J.; Barron, M. G.; Greene, R. M. Comparative Toxicity of Eight Oil Dispersants, Louisiana Sweet Crude Oil (LSC), and Chemically Dispersed LSC to Two Aquatic Test Species. Environ. Toxicol. Chem. 2011, 30, 2244-2252.

(16) Geitner, N. K.; Powell, R. R.; Bruce, T.; Ladner, D. A.; Ke, P. C. Effects of Dendrimer Oil Dispersants on Dictyostelium Discoideum. RSC Adv. 2013, 3, 25930.

(17) Petit, A.-N.; Debenest, T.; Eullaffroy, P.; Gagné, F. Effects of a Cationic PAMAM Dendrimer on Photosynthesis and ROS Production of Chlamydomonas Reinhardtii. Nanotoxicology 2012, 6, 315-326.

(18) Cancino, J.; Paino, I. M. M.; Micocci, K. C.; Selistre-de-Araujo, H. S.; Zucolotto, V. In Vitro Nanotoxicity of Single-Walled Carbon Nanotube-Dendrimer Nanocomplexes against Murine Myoblast Cells. Toxicol. Lett. 2013, 219, 18-25.

(19) Lewis, T.; Ganesan, V. Interactions between Grafted Cationic Dendrimers and Anionic Bilayer Membranes. J. Phys. Chem. B 2013, 117, 9806-9820. 
(20) Thomas, T. P.; Majoros, I.; Kotlyar, A.; Mullen, D.; Holl, M. M. B.; Baker, J. R. Cationic Poly(amidoamine) Dendrimer Induces Lysosomal Apoptotic Pathway at Therapeutically Relevant Concentrations. Biomacromolecules 2009, 10, 3207-3214.

(21) Jones, C. F.; Campbell, R. A.; Brooks, A. E.; Assemi, S.; Tadjiki, S.; Thiagarajan, G.; Mulcock, C.; Weyrich, A. S.; Brooks, B. D.; Ghandehari, H.; et al. Cationic PAMAM Dendrimers Aggressively Initiate Blood Clot Formation. ACS Nano 2012, 6, 9900-9910.

(22) Bhattacharya, P.; Geitner, N. K.; Sarupria, S.; Ke, P. C. Exploiting the Physicochemical Properties of Dendritic Polymers for Environmental and Biological Applications. Phys. Chem. Chem. Phys. 2013, 15, 4477-4490.

(23) Ciolkowski, M.; Petersen, J. F.; Ficker, M.; Janaszewska, A.; Christensen, J. B.; Klajnert, B.; Bryszewska, M. Surface Modification of PAMAM Dendrimer Improves Its Biocompatibility. Nanomedicine 2012, 8, 815-817.

(24) Maiti, P. K.; Li, Y.; Cagin, T.; Goddard, W. A. Structure of Polyamidoamide Dendrimers up to Limiting Generations: A Mesoscale Description. J. Chem. Phys. 2009, 130, 144902.

(25) Wu, B.; Kerkeni, B.; Egami, T.; Do, C.; Liu, Y.; Wang, Y.; Porcar, L.; Hong, K.; Smith, S. C.; Liu, E. L.; et al. Structured Water in Polyelectrolyte Dendrimers: Understanding Small Angle Neutron Scattering Results through Atomistic Simulation. J. Chem. Phys. 2012, 136, 144901.

(26) Wu, B.; Liu, Y.; Li, X.; Mamontov, E.; Kolesnikov, A. I.; Diallo, S. O.; Do, C.; Porcar, L.; Hong, K.; Smith, S. C.; et al. ChargeDependent Dynamics of a Polyelectrolyte Dendrimer and Its Correlation with Invasive Water. J. Am. Chem. Soc. 2013, 135, 5111-5117.

(27) Liu, Y.; Porcar, L.; Hong, K.; Shew, C.-Y.; Li, X.; Liu, E.; Butler, P. D.; Herwig, K. W.; Smith, G. S.; Chen, W.-R. Effect of Counterion Valence on the $\mathrm{pH}$ Responsiveness of Polyamidoamine Dendrimer Structure. J. Chem. Phys. 2010, 132, 124901.

(28) Maingi, V.; Kumar, M. V. S.; Maiti, P. K. PAMAM DendrimerDrug Interactions: Effect of $\mathrm{pH}$ on the Binding and Release Pattern. J. Phys. Chem. B 2012, 116, 4370-4376.

(29) Lin, S.-T.; Maiti, P. K.; Goddard, W. A. Dynamics and Thermodynamics of Water in PAMAM Dendrimers at Subnanosecond Time Scales. J. Phys. Chem. B 2005, 109, 8663-8672.

(30) Maiti, P. K.; Bagchi, B. Diffusion of Flexible, Charged, Nanoscopic Molecules in Solution: Size and $\mathrm{pH}$ Dependence for PAMAM Dendrimer. J. Chem. Phys. 2009, 131, 214901.

(31) Ding, F.; Tsao, D.; Nie, H.; Dokholyan, N. V. Ab Initio Folding of Proteins with All-Atom Discrete Molecular Dynamics. Structure 2008, 16, 1010-1018.

(32) Ding, F.; Dokholyan, N. V. Emergence of Protein Fold Families through Rational Design. PLoS Comput. Biol. 2006, 2, e85.

(33) Yin, S.; Biedermannova, L.; Vondrasek, J.; Dokholyan, N. V. MedusaScore: An Accurate Force Field-Based Scoring Function for Virtual Drug Screening. J. Chem. Inf. Model. 2008, 48, 1656-1662.

(34) Neria, E.; Fischer, S.; Karplus, M. Simulation of Activation Free Energies in Molecular Systems. J. Chem. Phys. 1996, 105, 1902.

(35) Lazaridis, T.; Karplus, M. Effective Energy Functions for Protein Structure Prediction. Curr. Opin. Struct. Biol. 2000, 10, 139-145.

(36) Andersen, H. C. Molecular Dynamics Simulations at Constant Pressure And/or Temperature. J. Chem. Phys. 1980, 72, 2384.

(37) Antosiewicz, J. M.; Shugar, D. Poisson-Boltzmann ContinuumSolvation Models: Applications to $\mathrm{pH}$-Dependent Properties of Biomolecules. Mol. Biosyst. 2011, 7, 2923-2949.

(38) Porcar, L.; Liu, Y.; Verduzco, R.; Hong, K.; Paul, D.; Magid, L. J.; Smith, G. S.; Chen, W.; Butler, P. D. Structural Investigation of PAMAM Dendrimers in Aqueous Solutions Using Small-Angle Neutron Scattering: Effect of Generation. J. Phys. Chem. B 2008, $112,14772-14778$.

(39) Porcar, L.; Hong, K.; Butler, P. D.; Herwig, K. W.; Smith, G. S.; Liu, Y.; Chen, W. Intramolecular Structural Change of PAMAM Dendrimers in Aqueous Solutions Revealed by Small-Angle Neutron Scattering. J. Phys. Chem. B 2010, 114, 1751-1756.
(40) Liu, Y.; Chen, C.-Y.; Chen, H.-L.; Hong, K.; Shew, C.-Y.; Li, X.; Liu, L.; Melnichenko, Y. B.; Smith, G. S.; Herwig, K. W.; et al. Electrostatic Swelling and Conformational Variation Observed in High-Generation Polyelectrolyte Dendrimers. J. Phys. Chem. Lett. 2010, 1, 2020-2024.

(41) Liu, Y.; Bryantsev, V. S.; Diallo, M. S.; Goddard, W. A. PAMAM Dendrimers Undergo $\mathrm{pH}$ Responsive Conformational Changes without Swelling. J. Am. Chem. Soc. 2009, 131, 2798-2799.

(42) Lee, H.; Larson, R. G. Molecular Dynamics Simulations of PAMAM Dendrimer-Induced Pore Formation in DPPC Bilayers with a Coarse-Grained Model. J. Phys. Chem. B 2006, 110, 18204-18211.

(43) Yang, L.; da Rocha, S. R. P. PEGylated, NH2-Terminated PAMAM Dendrimers: A Microscopic View from Atomistic Computer Simulations. Mol. Pharmaceutics 2014, 11, 1459-1470.

(44) Niu, S.; Turro, C.; Bossmann, S. H.; Tomalia, D. A.; Turro, N. J. Binding of $* \mathrm{Ru}($ phen $) 32+$ to Starburst Dendrimers and Its Quenching by Co(phen)33+: Generation Dependence of the Quenching Rate Constant. J. Phys. Chem. 1995, 99, 5512-5517. 


\section{The Structure-Function Relationship of PAMAM Dendrimers as Host-Guest Agents for Oil Dispersion}

Nicholas K Geitner, ${ }^{\dagger}$ Bo Wang, ${ }^{\dagger}$ Rachel E Andorfer, ${ }^{\dagger}$ David A Ladner, ${ }^{\ddagger}$ Pu Chun Ke, ${ }^{\dagger}$ and Feng Ding $^{\dagger^{* *}}$

Author Addresses

$\dagger$ Department of Physics and Astronomy, Clemson University, Clemson, SC 29634, USA

$\$$ Department of Environmental Engineering and Earth Sciences, Clemson University, Clemson, SC 29634, USA

*Corresponding Author. E-mail: fding@clemson.edu

\section{Supporting Information}

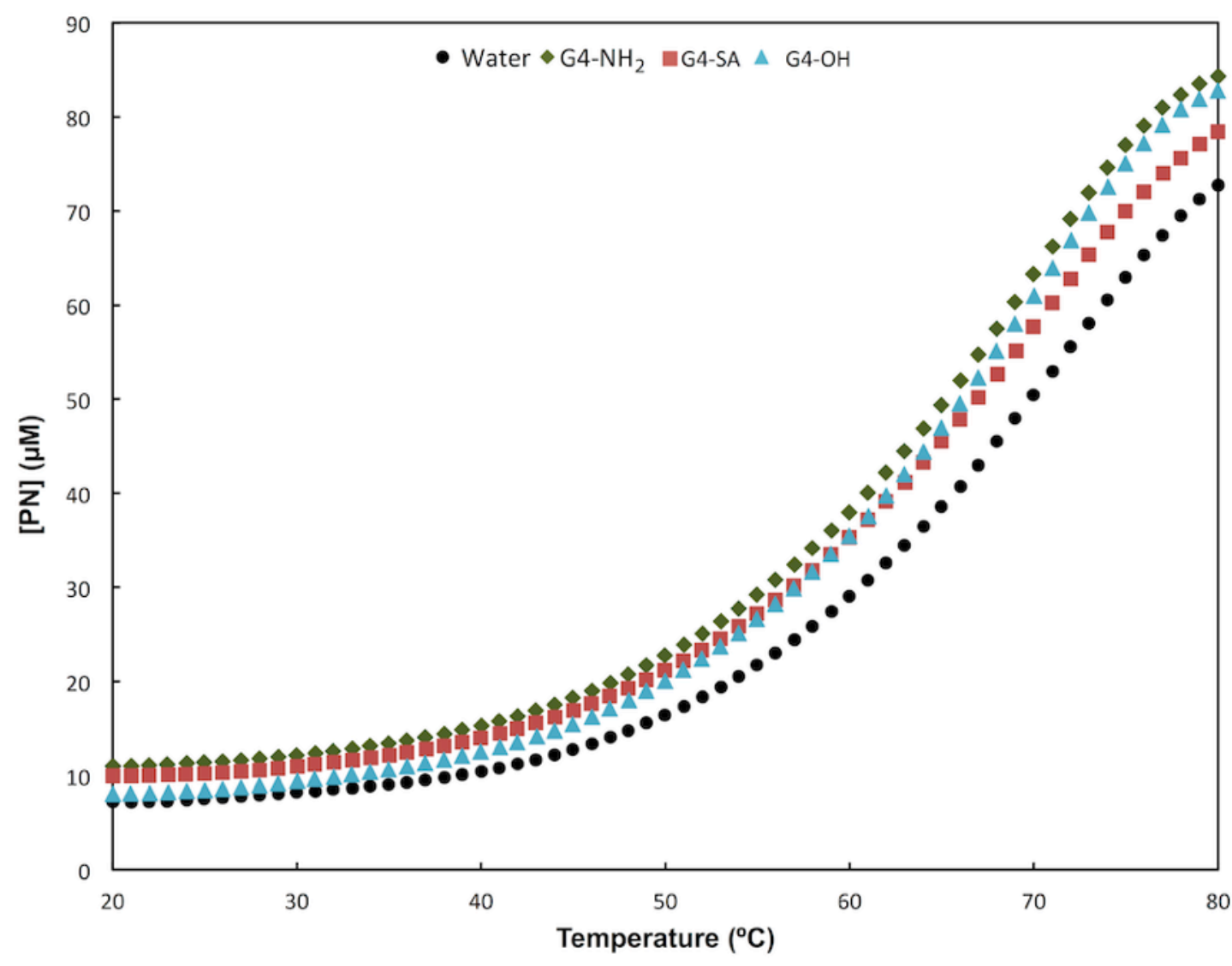

Figure S1. The total concentration of phenanthrene in water or dendrimer solutions as a function of temperature averaged over 3 independent trials for each solution. 


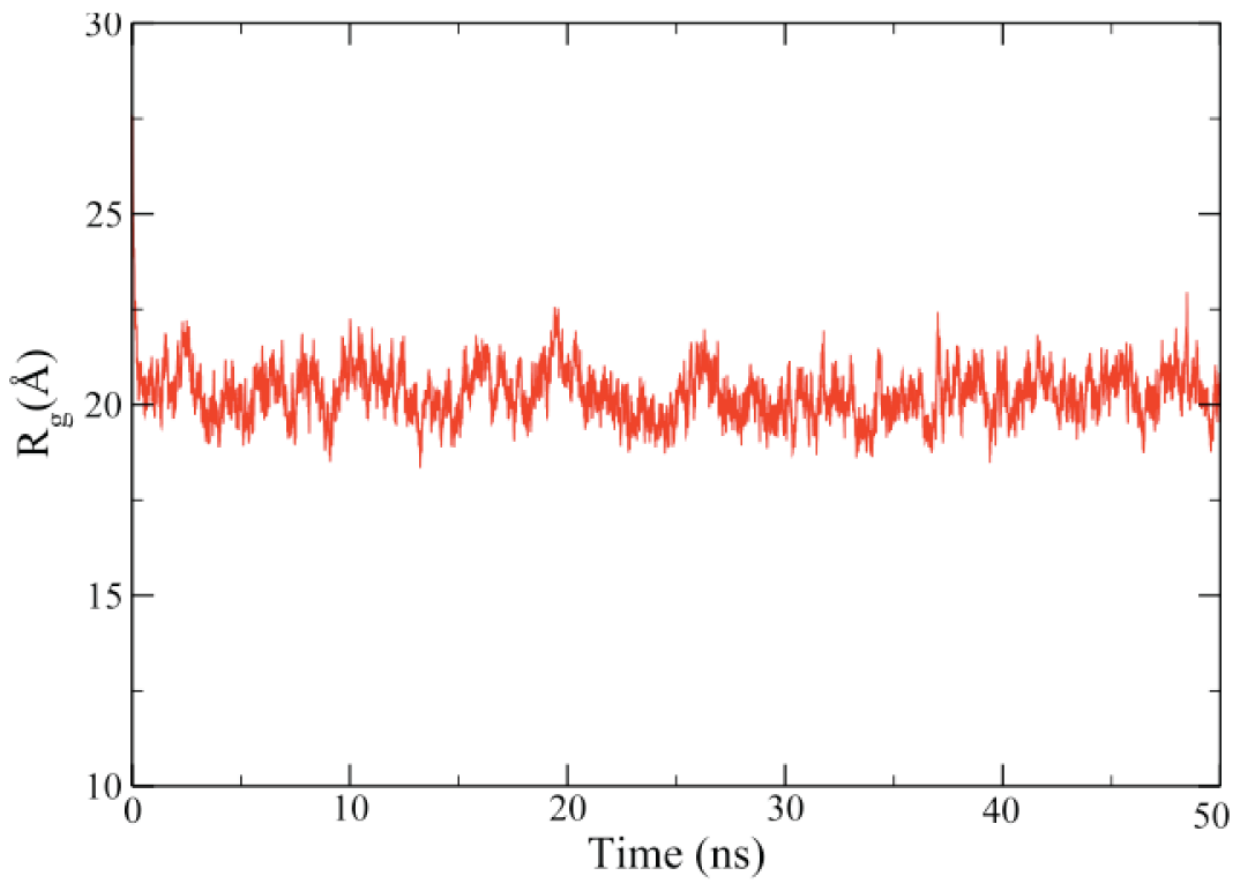

Figure S2. A representative simulation trajectory of $\mathrm{Rg}$ as function of time for a $\mathrm{G} 4-\mathrm{NH}_{2}$ dendrimer (neutral $\mathrm{pH}$ ), showing rapid relaxation (2 ns) and equilibration of the system with small thermal fluctuations thereafter. 


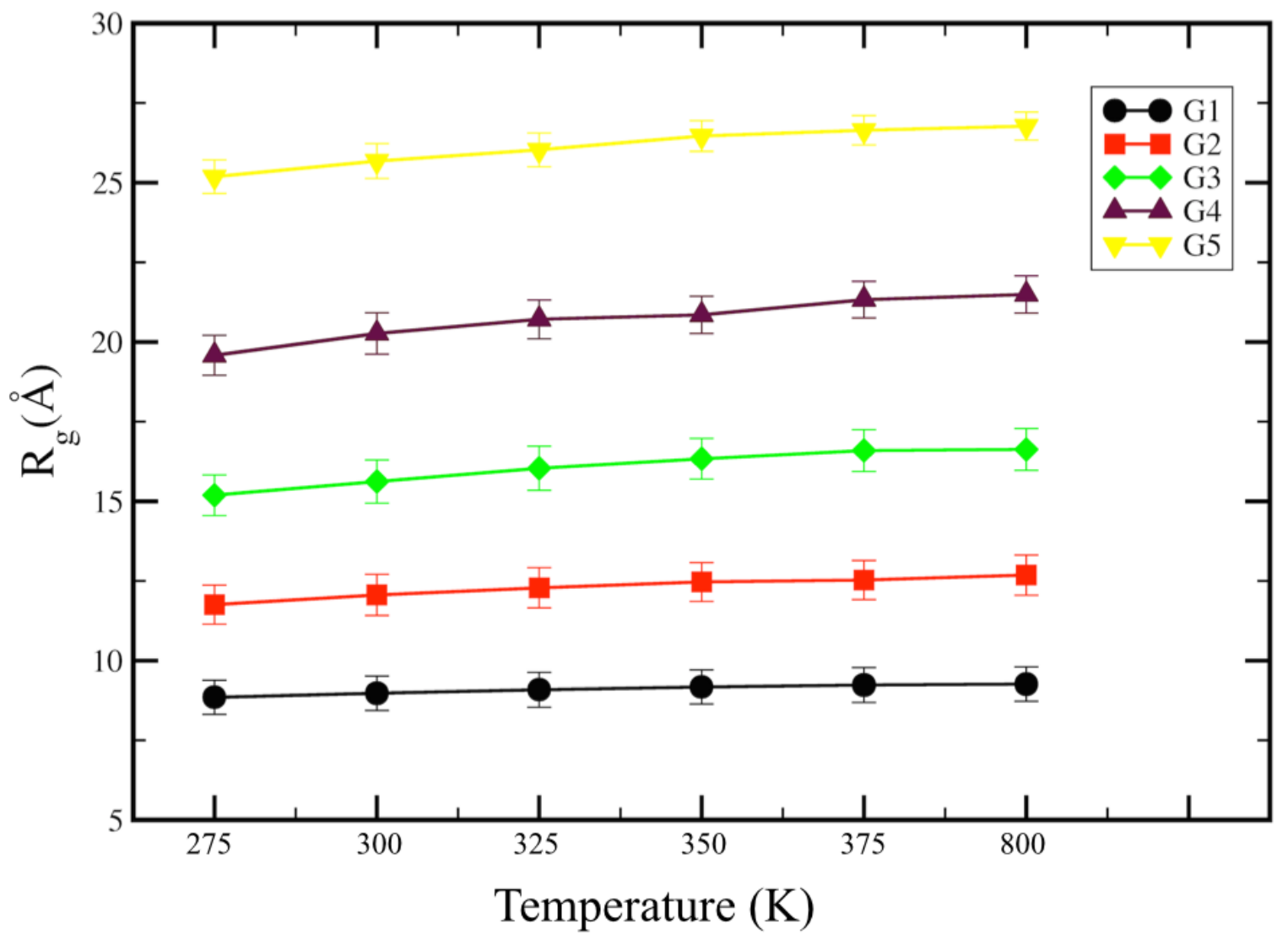

Figure S3. The radius of gyration of $\mathrm{NH}_{2}$ terminated PAMAM dendrimers of generation 1-5, as a function of temperature. Error bars correspond to standard deviations. 

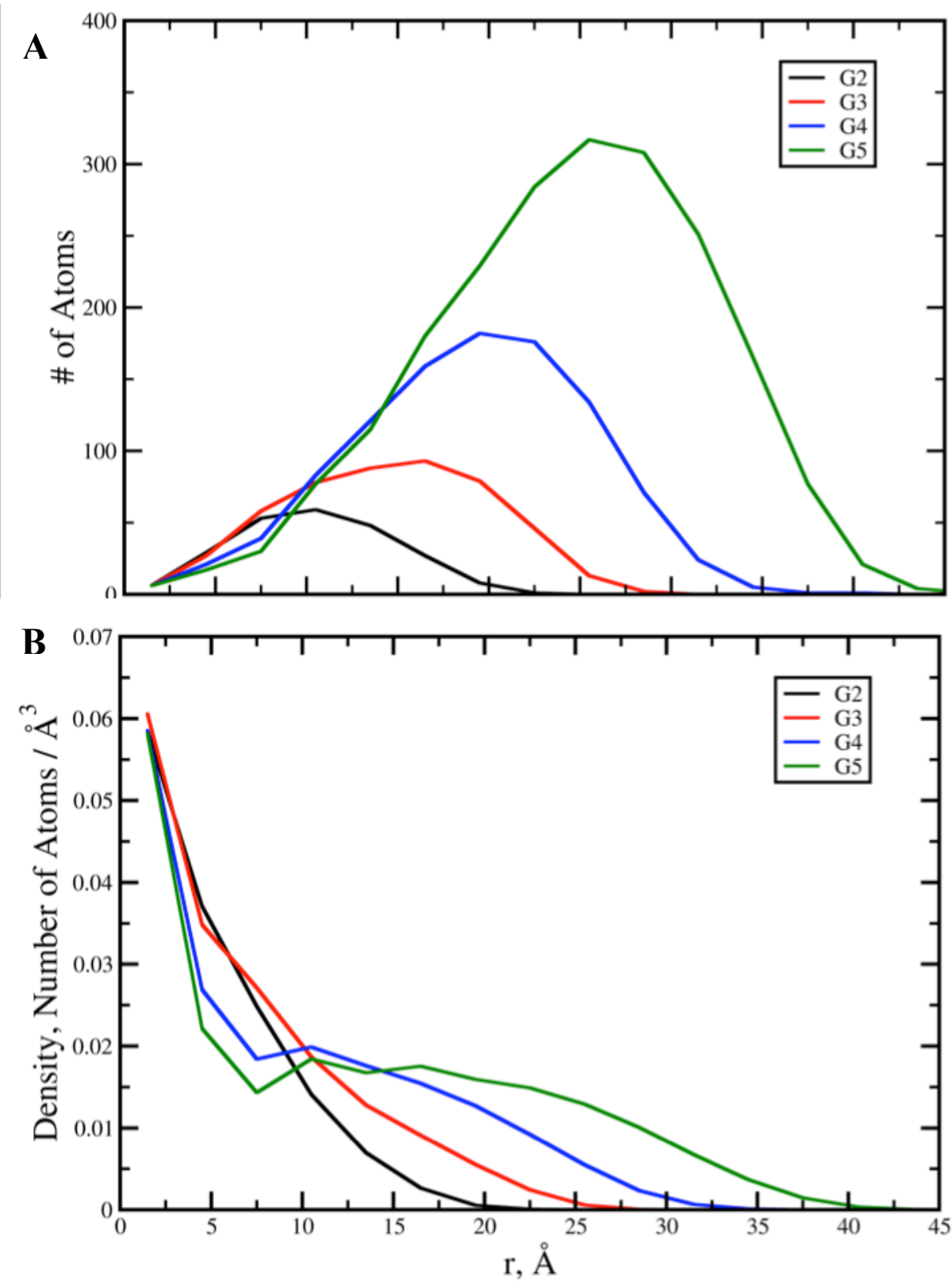

Figure S4. The radial atom distribution (A) and radial density distribution (B) functions are calculated for $\mathrm{NH}_{2}$-terminated dendrimers of various generations from G2 to G5. Density is computed as the number of atoms per unit volume. The functions are averaged over time where the molecular systems reach equilibrium. As the generation increases, the atom density near the core decreases. 


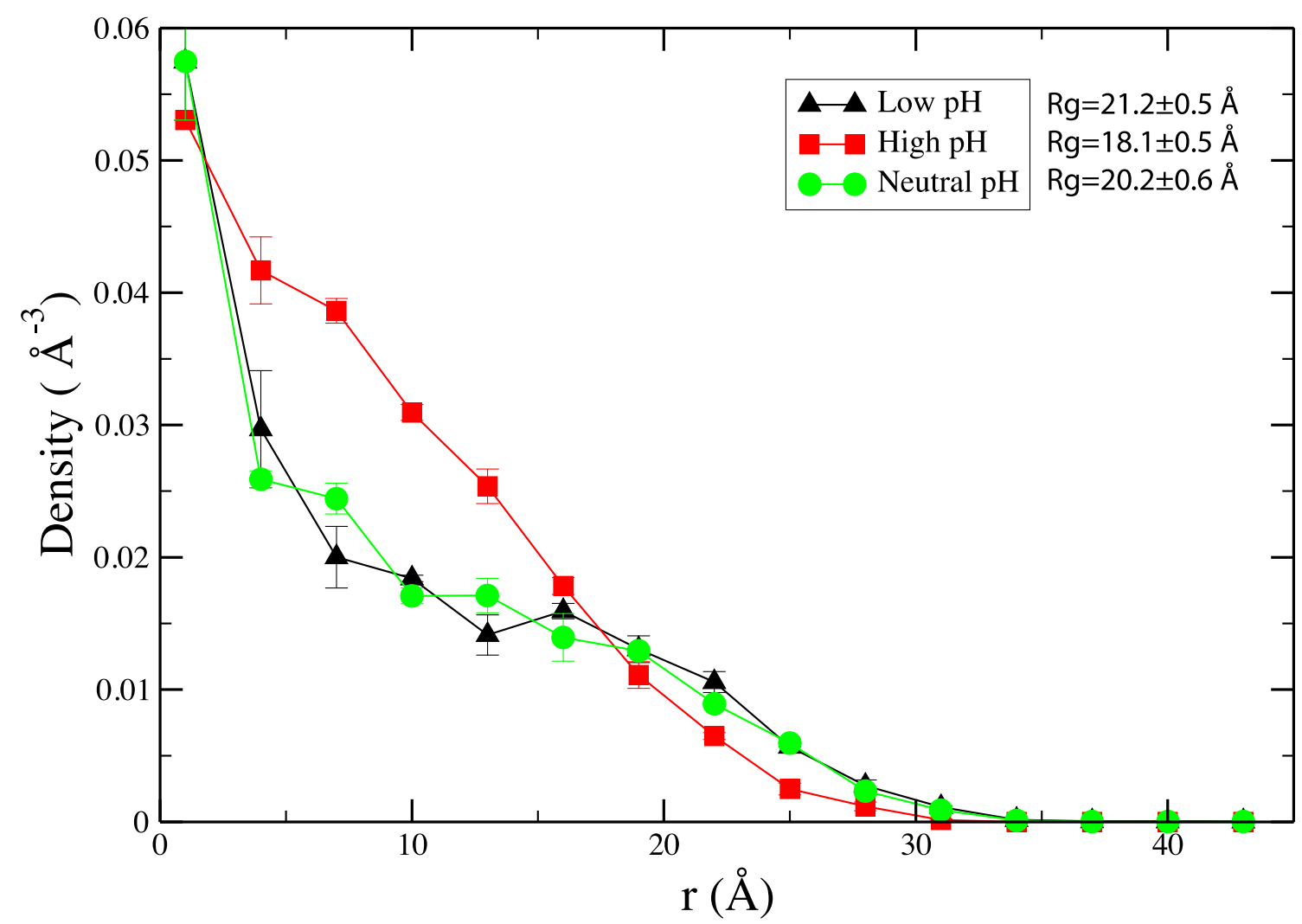

Figure S5. Radial density profile of G4- $\mathrm{NH}_{2}$ at low, neutral, and high $\mathrm{pH}$, defined as completely protonated, only primary amines protonated, and completely deprotonated, respectively. The error bars are calculated from two independent DMD simulations. We observe a transition from "dense core" $(<15 \AA)$ at high $\mathrm{pH}$ to "dense shell" $(>15 \AA)$ at neutral and low $\mathrm{pH}$, in agreement with previous MD simulations (1). The average $\mathrm{R}_{\mathrm{g}}$ values show modest changes in average size (see inset).

\section{References}

1. Liu, Y.; Bryantsev, V. S.; Diallo, M. S.; Goddard, W. A. PAMAM Dendrimers Undergo pH Responsive Conformational Changes without Swelling. J. Am. Chem. Soc. 2009, 131, 27982799 